\title{
Cost Allocation in Water Resources Development
}

\author{
H. P. Young ${ }^{1}$ \\ International Institute for Applied Systems Analysis, Laxenburg, Austria \\ N. OKADA \\ Tottori University, Koyama Tottori, Japan
}

T. Hashimoto

International Development Center of Japan. Tokyo, Japan

\begin{abstract}
Different methods for allocating the joint costs of water supply projects among users are compared on the basis of certain commonsense principles of equity. We contrast the separable costs-remaining benefits (SCRB) method with simple proportional allocation schemes and more sophisticated methods from cooperative game theory, including the Shapley value and variants of the core. Advantages and disadvantages of the methods in practice are examined using a regional water supply system in Sweden. It is argued that these principles provide a useful framework for choosing intelligently among methods. The appropriateness of a method depends on the context, especially on the reliability of information about costs and demands. The conclusion is that there is no one best method, although from a normative standpoint the SCRB method may be one of the worst.
\end{abstract}

\section{INTRODUCTION}

A central problem in planning the provision of goods or services by a public enterprise is how to determine a 'fair' or 'just' allocation of joint costs. This problem is particularly acute in the development of a common water resource like a multipurpose reservoir, where the greater cost-effectiveness of larger facilities means that the sum of the marginal costs of each participant may be much less than the total cost of the project. The literature on water resources contains a variety of proposals for dealing with this kind of problem, some of which anticipate ideas (like the core) later developed in game theory [Ransmeier, 1942; see also Loughlin, 1977; Heaney, 1979; Straffin and Heaney; 1981].

The methods most commonly used in current water resource planning practice are (1) to allocate costs in proportion to some single numerical criterion, such as use, population, or level of benefit, or (2) to allocate certain costs (e.g., marginal costs) directly, and divide the remainder on the basis of some scheme similar to the first method. Chief among the methods of the second type is the so-called separable costs-remaining benefits (SCRB) method, which is used for multipurpose reservoir projects in the United States [Inter-Agency Committee on Water Resources, 1958; Eckstein, 1958] and in other countries. This method will be treated in more detail in section 6.

The same type of problem has also been extensively treated in the economics literature on public utility pricing. An approach commonly advocated there is Ramsey pricing [Ramsey, 1927], which is based on the idea of setting prices to maximize some criterion of economic efficiency, e.g.,

\footnotetext{
${ }^{1}$ Now at the School of Public Affairs, University of Maryland, College Park, Maryland 20742.

Copyright 1982 by the American Geophysical Union.

Paper number 1W1852.

0043-1399/82/001W-1852\$05.00
}

consumer surplus, subject to a breakeven constraint [Baumol and Bradford, 1970]. This approach relies on having extensive information about the demand for the goods and services over a range of consumption levels-information that is often not available in practice, especially for a resource like water in which established markets may not even exist. Ramsey pricing is also less suitable for planning long-term investments, where future demand can only be estimated.

A third strand in the literature comes from the theory of cooperative games, which provides various normative approaches to the problem of allocating joint costs (and benefits) among users by taking the strategic possibilities into account. Among the most commonly used of these game theory concepts are the Shapley value and the nucleolus. The application of these ideas to water resource projects is increasing; for a sample of the recent literature see Loehman and Whinston [1974], Suzuki and Nakayama [1976], Bogardi and Szidarovsky [1976], Okada [1977], and Loehman et al. [1979]. Methods from the theory of cooperative games are particularly well suited to contexts like water resources development, in which the basic decision is often whether or not to supply a potential class of users at some targeted level, the implicit assumption being that this level is close to the optimal scale of development. The advantage is that the analysis is not made to depend on precise estimations of future demand curves, but only on 'point' estimates of benefits. The disadvantage is that even such point estimates are often unreliable; moreover, it is implicitly assumed in the analysis that the optimal scale of development is known, which ultimately presumes that demands as well as costs are known. It has recently been shown that these shortcomings may be overcome by designing an appropriate noncooperative game, which reveals both consumer demands and the optimal scale of development and at the same time allocates costs in a manner consistent with principles of cooperative equity [Young, 1980]. 
The complexity of the cost allocation problem has led some authors to conclude that there is no economically justifiable way to allocate joint costs [see Ransmeier, 1942; Thomas, 1974]. Nevertheless, in practice the costs of a project must usually be allocated in some way among the beneficiaries. The purpose of this paper is to compare the merits of traditional methods of allocating costs with several well known and other lesser known methods from cooperative game theory. The analysis consists of two parts. First, we discuss certain established principles of 'fairness' by which the different methods can be judged. Second, we compare the solutions given by each method for an actual example: a cost-sharing problem among a group of municipalities in Sweden who wish to develop a joint municipal water supply. The object of this analysis is not to provide a strict axiomatic characterization of methods but rather to explore their behavior in practice. Our conclusion is that of the traditional approaches, proportional allocation according to a single numerical criterion may be preferable to the more complicated SCRB method, provided that the proportionality criterion seems fair and the accuracy of the values is not open to dispute. The SCRB method is shown to be seriously flawed in that it is not monotonic in total costs: that is, an increase in total costs may result in some participants having to pay less than before; this is due to the ad hoc way in which marginal costs are introduced. The game theory methods require more information and are more complicated; moreover, one of the best known of these methods, the nucleolus, is also not monotonic in total costs. By contrast a lesser known variation of the nucleolus, the proportional nucleolus, passes most of the tests of fairness considered here and would appear to merit serious consideration for cost allocation in water resources development.

\section{Joint Cost Functions}

Consider three neighboring municipalities, A, B, and C, who can supply themselves with municipal water either by building separate facilities or by building a joint water supply facility. We suppose that the joint facility is cheaper to construct than the separate projects due to economies of scale. The quantity of water to be supplied to each municipality is assumed given. The problem is then how to divide the costs among them.

Table 1 shows the population of each municipality, the amounts of water to be supplied in each, and the unit costs of building separate facilities for each. Table 2 shows the costs of supplying the target amounts for different combinations. All possibilities are considered: $A$ and $B$ build a joint facility and $C$ goes alone; $B$ and $C$ cooperate and $A$ goes alone, and so on.

The cost figures show that building a facility to serve all three communities will be $1.6 \times 10^{6}$ dollars cheaper than if three separate facilities were built. However, A and B together can also realize savings of $\$ 400,000$ without including $A$. A and C, being geographically separated by $B$, cannot

TABLE 1. Annual Rates of Water Use and Unit Costs of Supplying Water in Three Hypothetical Municipalities

\begin{tabular}{ccc}
\hline Municipality & $\begin{array}{c}\text { Use per Capita, } \\
\mathrm{m}^{3}\end{array}$ & $\begin{array}{c}\text { Unit Cost, } \\
\text { dollars } / \mathrm{m}^{3}\end{array}$ \\
\hline A & 140 & 0.46 \\
B & 120 & 0.88 \\
C & 120 & 1.25 \\
\hline
\end{tabular}

TABLE 2. Annual Costs of Water Supply Under Various Combinations

\begin{tabular}{lcc}
\hline Combination & $\begin{array}{c}\text { Cost Breakdown, } \\
\text { dollars } \times 10^{6}\end{array}$ & $\begin{array}{c}\text { Total Cost, } \\
\text { dollars } \times 10^{6}\end{array}$ \\
\hline $\mathrm{A}+\mathrm{B}+\mathrm{C}$ & $6.5+4.2+1.5$ & 12.2 \\
$\mathrm{~A}+\{\mathrm{B}, \mathrm{C}\}$ & $6.5+5.3$ & 11.8 \\
$\{\mathrm{~A}, \mathrm{~B}\}+\mathrm{C}$ & $10.3+1.5$ & 11.8 \\
$\{\mathrm{~A}, \mathrm{C}\}^{*}+\mathrm{B}$ & $8.0+4.2$ & 12.2 \\
$\{\mathrm{~A}, \mathrm{~B}, \mathrm{C}\}$ & 10.6 & 10.6 \\
\hline
\end{tabular}

$*\{\mathrm{~A}, \mathrm{C}\}=\mathrm{A}+\mathrm{C}=\mathbf{8 . 0}$.

do any better by building a joint facility than they could by building separate facilities. The most cost-effective way of supplying water would be to build a joint facility serving all three communities.

More generally, let $\{1,2, \cdots, n\}=N$ represent a group of prospective participants in a cooperative venture to provide a product or service to members of the group. The cost of serving a subgroup $S$, denoted by $c(S)$, is found by considering the least cost alternative of providing the same service, either jointly or singly, to the members of $S$ independently of how the others are served. The joint cost function $c(S)$ so defined must be subadditive, i.e., must satisfy $c(S)+c(T)>$ $c(S \cup T)$ for any two nonoverlapping groups $S$ and $T$, because the ways of serving $S$ together with $T$ include the possibility of serving $S$ alone and $T$ alone. Thus in the above example, municipalities $\mathrm{A}$ and $\mathrm{C}$ would find it quite costly to build a single joint facility because of their geographical separation; hence the least cost alternative would be to build one facility for $\mathrm{A}$ and another for $\mathrm{C}$.

If the cost of serving any group of users is simply the sum of the costs of serving them singly, then the cost allocation problem is trivial. The more interesting and more typical case arises when the cost of serving several users by some joint facility is less than the sum of serving them singly, that is, $c(N)<\Sigma_{N} c(i)$. The cost savings that would result from cooperating in a coalition $S$ instead of going alone are

$$
v(S)=\sum_{S} c(i)-c(S) \geq 0
$$

The function $\nu$ is called the cost savings game. To illustrate the different situations that can arise, suppose that the cost of serving a group $S$ depends only on the number of members of $S$ and that the cost savings are greatest for larger groups due to economies of scale. One possibility is that the cost savings increase at a greater rate with each additional participant. Another, perhaps more typical situation is that the rate of increase first rises, then falls, as shown in Figure 1. Justifying a fair allocation of costs is considerably more difficult in the latter case than in the former.

\section{Principles of Equity: The Core}

'Equity' can have a wide range of meanings. When considering what it means for a cost allocation to be equitable, it is first helpful to ask ourselves why it should be equitable. The answer is, to encourage the agreement or cooperation of those who pay the costs. Thus a reasonable test of the equity of a method is to see whether the participants agree in principle to the proposed allocation of costs.

In practice one of the most common ways of achieving agreement on the distribution of costs is simply to allocate the costs in proportion to a criterion which stands for 


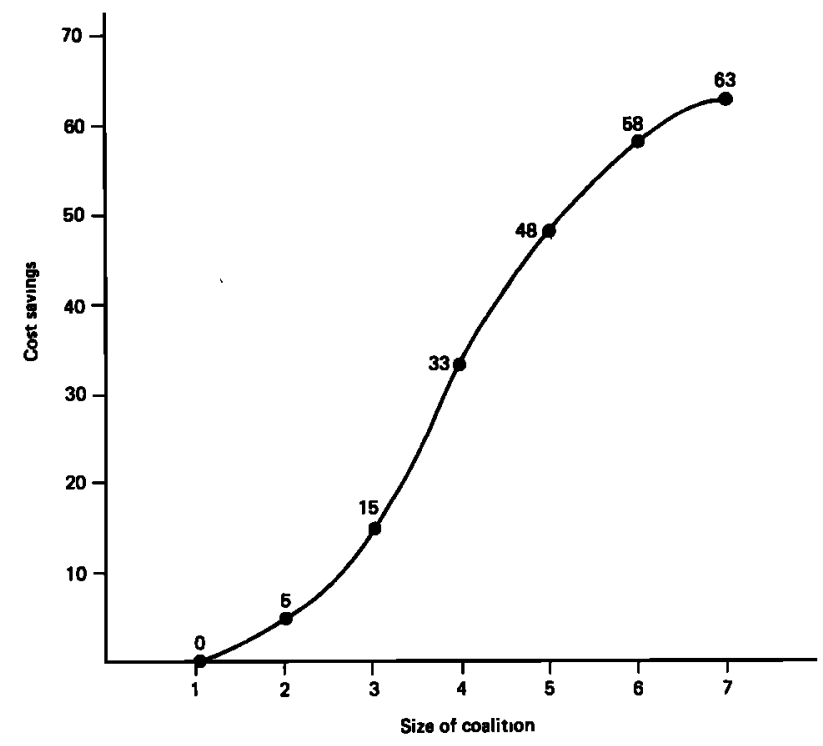

Fig. 1. Cost savings as a function of the size of coalitions.

benefits received, such as use of facilities, number of users, or indeed, the benefits themselves-if they can be reliably estimated. Ersatz measures of benefits may actually be the more persuasive simply because their magnitudes are less open to dispute. This is the case in the municipal cost sharing problem discussed in section 7 . Here the costs to be shared relate to long-term investments and future demand is conjectural at best, so the populations of the municipalities have been used as the basis for allocation.

One difficulty with allocating costs in this way is that it may conflict with some participants' perceptions of selfinterest and hence may not provide sufficient incentive for cooperation. In the municipality example above, an allocation of costs according to populations would result in the shares (in millions of dollars) 7.067 for $\mathrm{A}, 2.827$ for $\mathrm{B}$, and 0.707 for $C$. However, municipality $A$ would find it hard to accept such an allocation, since it could provide the same amount of water on its own for only $\$ 6.5$ million. An allocation made on the basis of use gives the shares 7.420 for A, 2.544 for $B, 0.636$ for $C$. A is even worse off by this allocation.

The difficulty with 'proportional' allocation methods is that they ignore one of the fundamentals of the problem: the alternative costs embodied in the joint cost function $c(S)$. It is a minimum requirement of a fair allocation that it be individually rational: no user should pay more in the joint venture than he would have to pay on his own. This principle was stated by Ransmeier [1942] for the Tennessee Valley Authority projects and is well established in the game theory literature [von Neumann and Morgenstern, 1944]. It is a fundamental concept of fairness, since it constitutes the minimum incentive for an individual to join. To be individually rational, the costs $\mathrm{y}$ allocated to $\mathrm{A}, \mathrm{B}$, and $\mathrm{C}$ in the above example must satisfy $y_{A} \leq 6.5, y_{B} \leq 4.2, y_{C} \leq 1.5$, where $y_{\mathrm{A}}+y_{\mathrm{B}}+y_{\mathrm{C}}=\$ 10.6$ million.

The same argument can be applied to groups of participants as well as to individuals and indeed was so stated by Ransmeier [1942]. Consider, for example, neighboring municipalities $A$ and $B$ : they can build a joint facility for $\$ 10.3$ million, so it would certainly be unfair to allocate them more than $\$ 10.3$ million in total costs. Not only would it be unfair, but also, if cooperation is voluntary, there is a risk of $A$ and $B$ backing out if they have an allocation $y_{A}+y_{B}>10.3$, since they can do better as a pair. The condition that no group pay more than its alternative cost is the principle of group rationality. Since a group may consist of a single participant, group rationality implies individual rationality.

In the general case, where there are $n$ independent users $\{1,2, \cdots, n\}=N$ and alternative costs are given by the function $c(S)$, the condition for group rationality of a cost allocation $\mathbf{y}=\left(y_{1}, y_{2}, \cdots, y_{n}\right), \Sigma_{N} y_{i}=c(N)$, is that

$$
\sum_{S} y_{i} \leq c(S) \quad \forall S \subset N
$$

A second principle with firm roots in the economics and project evaluation literature is that no participant should be charged less than the marginal cost (sometimes called the separable cost) of including him in the project [Federal InterAgency River Basin Committee, 1950; Inter-Agency Committee on Water Resources, 1958; Water Resources Council, 1962 ]. For example, it costs $\$ 10.6$ million to serve $A, B$, and $\mathrm{C}$ but only $\$ 8.0$ million to serve $\mathrm{A}$ and $\mathrm{C}$. Therefore the marginal (or separable) cost of serving $B$ is $\$ 10.6-\$ 8.0=$ $\$ 2.6$ million, so it seems only fair that $B$ should pay at least this amount. This concept is called individual marginal cost coverage.The marginal costs for $\mathrm{A}, \mathrm{B}$, and $\mathrm{C}$ are $\$ 5.3,2.6$, and 0.3 million, respectively.

There is an obvious extension of this idea to groups. For example, the marginal cost of including the group $\{A, B\}$ is $\$ 10.6-\$ 1.5=\$ 9.1$ million. The principle of marginal cost coverage states that every group of users should be charged at least the additional cost of serving them. This is only fair, since if $y_{A}+y_{B}<9.1$, then $C$ would be subsidizing $A$ and $B$. The idea of finding prices in which users do not subsidize each other in the use of a given public service or facility is also well known in the theory of regulated public utilities [Faulhaber, 1975; Zajac, 1978].

Stated in general terms, the marginality principle is that a cost allocation y should satisfy

$$
\sum_{S} y_{i} \geq c(N)-c(N-S) \quad \forall S \subset N
$$

The argument for group rationality is based on strategic considerations, i.e., providing sufficient incentive to prevent users from withdrawing, while marginality can be viewed as a general concept of fairness that can be applied even if cooperation is mandated. However, an inspection of these two ideas reveals that they are in fact equivalent, given that all costs must be allocated, i.e., that $\Sigma_{N} y_{i}=c(N)$.

In game theory it is customary to interpret the two equivalent conditions (1) and (2) in terms of the cost savings game $v$. Any allocation $\mathbf{y}$ of costs implies a corresponding imputation $\mathrm{x}$ of savings: if $y_{i}$ is the cost assessed for $i$, then the amount $i$ saves by cooperating rather than going alone, $x_{i}$, is given by $y_{i}=c(i)-x_{i}$. In terms of $v$, the condition of individual rationality says that $x_{i} \geqq 0$ for every participant $i$; group rationality says that

$$
\begin{aligned}
& \sum_{S} x_{i} \geqq v(S) \quad \forall S \subset N \\
& \sum_{N} x_{i}=v(N)
\end{aligned}
$$


The set of all vectors $\mathbf{x}$ satisfying (3) is called the core of the game $v$.

In our example the core is the set of solutions to the following inequalities:

$$
\begin{aligned}
\mathrm{x} & \geqq 0 \\
x_{A}+x_{B} & \geqq v(\mathrm{~A}, \mathrm{~B})=(6.5+4.2)-10.3=0.4 \\
x_{A}+x_{C} & \geqq v(\mathrm{~A}, \mathrm{C})=(6.5+1.5)-8.0=0.0 \\
x_{\mathrm{B}}+x_{\mathrm{C}} & \geqq v(\mathrm{~B}, \mathrm{C})=(4.2+1.5)-5.3=0.4 \\
x_{\mathrm{A}}+x_{\mathrm{B}}+x_{\mathrm{C}} & =v(\mathrm{~A}, \mathrm{~B}, \mathrm{C})=(6.5+4.2+1.5)-10.3=1.6
\end{aligned}
$$

The core provides a guideline for cost allocation by narrowing down the class of acceptable imputations, but it does not usually identify a unique answer. However, there is always the unfortunate possibility that there may be no core imputations; that is, no cost allocations that are either group rational or satisfy marginality. That this can happen in perfectly reasonable situations may be seen from Figure 1. Here there are increasing returns to scale, but the rate of increase first rises, then falls. The minimum savings that can be realized by all seven users together is 63 , but no matter how these savings are distributed, some group of five will receive at most 45 , even though they could save more (i.e., 48) as a subcoalition.

In such an example a quick test to see whether the core is empty is to draw a line from the origin to the point corresponding to the total number of users; this line segment must lie above the savings curve for the core to be nonempty [Shapley and Shubik, 1973]. In more complicated examples the feasibility of condition (3) can be checked using linear programing. In the three-municipality example the core is small; if the total cost $c(\mathrm{~A}, \mathrm{~B}, \mathrm{C})$ had happened to be $\$ 12.0$ million instead of $\$ 10.6$ million, the core would have been empty. Thus core imputations are not bound to exist; however, the greater the economies of scale, the more likely core imputations are to be present. Moreover, when core imputations exist, they are typically not unique.

\section{Methods Extending the Core}

One approach to resolving the twin difficulties of nonexistence and nonuniqueness has been to look for some natural way of strengthening (or relaxing) the inequalities defining the core. This is one of the most common approaches in the game theory literature. Three such methods will be discussed here: the least core and its specialization, the nucleolus, and two variations: the weak least core and weak nucleolus, and the proportional least core and proportional nucleolus.

\subsection{The Least Core and Nucleolus}

If the core of the cost savings game $v$ is empty, the best alternatives of some subgroups are very good-in a certain sense 'too' good-relative to the best alternative of the whole group. Hence we might consider imposing a tax on all proper subgroups as a way of encouraging the whole group to stick together. The least core is found by imposing the smallest uniform $\operatorname{tax} \varepsilon$ such that if all coalitions other than the whole group pay this tax, then a core imputation exists. Thus we require the least $\varepsilon$ for which there exists an imputation $\mathbf{x}$ satisfying

$$
\begin{aligned}
& \sum_{S} x_{i} \geq v(S)-\varepsilon \quad \forall S \subset N \\
& \sum_{N} x_{i}=v(N)
\end{aligned}
$$

The least core is the set of all imputations $\mathbf{x}$ satisfying (5) for this least $\varepsilon$ [Shapley and Shubik, 1973]. The corresponding allocations of costs are found by letting $y_{i}=c(i)-x_{i}$ for all $i$. Because of our assumptions about $v$, it can be shown that the result is always individually rational, i.e., that $x \geqq 0$.

Suppose on the other hand, that the cost savings game $v$ already has a core, and it is necessary to find a unique solution. One way of narrowing down the choice is to imagine subsidizing all coalitions other than the whole group by a uniform amount $\varepsilon$. This amounts to solving (5) for smallest $\varepsilon$ and allowing $\varepsilon$ to go negative.

Computing the least core involves solving a linear program. For the three-municipality example, this program (in millions of dollars) is:

$$
\min \varepsilon
$$

subject to

$$
\begin{gathered}
x_{\mathrm{A}} \geq-\varepsilon \quad x_{\mathrm{B}} \geq-\varepsilon \quad x_{\mathrm{C}} \geq-\varepsilon \\
x_{\mathrm{A}}+x_{\mathrm{B}} \geq 0.4-\varepsilon \\
x_{\mathrm{A}}+x_{\mathrm{C}} \geq-\varepsilon \\
x_{\mathrm{B}}+x_{\mathrm{C}} \geq 0.4-\varepsilon \\
x_{\mathrm{A}}+x_{\mathrm{B}}+x_{\mathrm{C}}=1.6
\end{gathered}
$$

The solution is $\varepsilon=-0.533, x_{\mathrm{A}}=x_{\mathrm{B}}=x_{\mathrm{C}}=0.533$, and the corresponding unique cost allocation is $y_{\mathrm{A}}=5.967, y_{\mathrm{B}}=$ 3.667, and $y_{\mathrm{C}}=0.967$.

In some cases the linear program (5) may have several solutions. If so, the following 'tie-breaking' device may be used. For any imputation $\mathrm{x}=\left(x_{1}, x_{2}, \cdots, x_{n}\right)$ and coalition $S$, define the excess of $S$ to be $v(S)-\Sigma_{S} x_{i}$. Let $e_{1}(\mathbf{x})$ be the largest excess of any coalition relative to $x, e_{2}(x)$ the second largest excess, $e_{3}(x)$ the next, and so on. The least core is the set $X_{1}$ of all $\mathrm{x}$ that minimize $e_{1}(x)$. Let $X_{2}$ be the set of all $\mathrm{x}$ in $X_{1}$ that minimize $e_{2}(\mathrm{x}), X_{3}$ the set of all $\mathrm{x}$ in $X_{2}$ that minimize $e_{3}(x)$, and so on. This process eventually leads to an $X_{k}$ consisting of a single imputation $\overline{\mathbf{x}}$, called the nucleolus [Maschler et al., 1979; Schmeidler, 1969].

\subsection{The Weak Least Core and Weak Nucleolus}

Suppose that a minimum uniform tax is imposed on any individual user who takes some course of action other than joining the whole group. Thus we find the least $\varepsilon$ for which there is a solution $x$ to the system:

$$
\begin{aligned}
& \sum_{S} x_{i} \geq v(S)-\varepsilon|S| \quad \forall S \subset N \\
& \sum_{N} x_{i}=v(N)
\end{aligned}
$$

where $|S|$ denotes the cardinality of the set $S$. The set of all corresponding imputations $\mathrm{x}$ is the weak least core. (For arbitrary $\varepsilon$ the set of all solutions to (7) has been called the 
'weak $\varepsilon$ core' [Shapley and Shubik, 1973]; hence the designation 'weak least core' in this case.) For the above example the weak least core is calculated by solving the linear program (in millions of dollars)

\section{$\min \varepsilon$}

subject to

$$
\begin{gathered}
x_{\mathrm{A}} \geq-\varepsilon \quad x_{\mathrm{B}} \geq-\varepsilon \quad x_{\mathrm{C}} \geq-\varepsilon \\
x_{\mathrm{A}}+x_{\mathrm{B}} \geq 0.4-2 \varepsilon \\
x_{\mathrm{A}}+x_{\mathrm{C}} \geq-2 \varepsilon \\
x_{\mathrm{B}}+x_{\mathrm{C}} \geq 0.4-2 \varepsilon \\
x_{\mathrm{A}}+x_{\mathrm{B}}+x_{\mathrm{C}}=1.6
\end{gathered}
$$

The unique solution is $\varepsilon=-0.4, x_{\mathrm{A}}=0.4, x_{\mathrm{B}}=0.8, x_{\mathrm{C}}=$ 0.4 , and the corresponding allocation of costs is $y_{A}=6.1, y_{B}$ $=3.4$, and $y_{\mathrm{C}}=1.1$.

A choice between multiple solutions can be made as in the computation of the nucleolus by defining the excess of $S$ to be $\left\{v(S)-\Sigma_{S} x_{i}\right\} /|S|$. We call the result the weak nucleolus. This method, while superficially similar to the least core, turns out to have an important property not shared by the least core (section 8). However, it also has drawbacks: in particular, it may not be individually rational when the core is empty. While individual rationality may simply be imposed as a constraint (as proposed by Grotte [1970, 1976]), another serious difficulty remains. The weak nucleolus may imply payments to dummies: partners who because of, say, geographical isolation, contribute nothing to the cost savings of any coalition. The dummy principle says that partners who contribute nothing to savings should go alone. The weak nucleolus violates this principle (a fact first noted by Reinhard Selten).

\subsection{The Proportional Least Core and Proportional Nucleolus}

Another variation on this theme is to modify the core by imposing a minimum tax (or subsidy) on all coalitions in proportion to their cost. Thus we postulate a tax rate $t$ and solve the system

$$
\min t
$$

subject to

$$
\begin{aligned}
& \sum_{S} x_{i} \geq(1-t) v(S) \quad \forall S \subset N \\
& \sum_{N} x_{i}=v(N)
\end{aligned}
$$

A minimum $t$ exists provided that $v(S)>0$ for some $S \neq N$. A choice between multiple solutions can be made, as in the computation of the nucleolus, by defining the excess of a coalition $S$ to be $\left\{v(S)-\Sigma_{S} x_{i}\right\} / v(S)$. (If $v(S)=0$, we adopt the convention that $a / 0>b / 0$ if $a>b$.)

For the above example the linear program (9) becomes

$$
\min t
$$

subject to

$$
\begin{gathered}
x \geq 0 \\
x_{\mathrm{A}}+x_{\mathrm{B}} \geq 0.4(1-t)
\end{gathered}
$$

$$
\begin{gathered}
x_{\mathrm{A}}+x_{\mathrm{C}} \geq 0 \\
x_{\mathrm{B}}+x_{\mathrm{C}} \geq 0.4(1-t) \\
x_{\mathrm{A}}+x_{\mathrm{B}}+x_{\mathrm{C}}=1.6
\end{gathered}
$$

The unique solution is $t=-3, x_{\mathrm{A}}=0, x_{\mathrm{B}}=1.6, x_{\mathrm{C}}=0$, and the corresponding cost allocation is $y_{A}=6.5, y_{B}=2.6$, and $y_{\mathrm{C}}=1.5$.

In other words, all of the savings in costs are allocated to B. The explanation for this is that $B$ has effective veto power; neither $\mathrm{A}$ nor $\mathrm{C}$ can do any better than going alone without forming a coalition that includes B. For other solution concepts related to the core, see Charnes et al. [1978], Maschler et al. [1979], and Heaney [1979].

\section{The Shapley Value}

The Shapley value for $n$ players is given by the formula

$y_{i}=\sum_{s=1}^{n} \frac{(s-1) !(n-s) !}{n !} \sum_{\substack{S: i \in S \\|S|=s}}[c(S)-c(S-i)]$

This is one of the earliest methods of allocation to be based on a consistent set of postulates about how an allocation should be made [Shapley, 1953]. All players are assumed to 'sign up' in some particular order. If a group $S$ has already signed up and $i$ was the last member of the group to arrive, his marginal cost contribution to $S$ is $c(S)-c(S-$ $i)$. The Shapley value is $i$ 's average marginal contribution if all orders for signing up are assumed to be equally likely.

The Shapley values for municipalities $A, B$, and $C$ are calculated in Table 3 . The first column shows the six possible orders for signing up; the next three columns show the marginal contribution of each municipality.

\section{The Separable Costs-Remaining Benefits (SCRB) METHOD}

The last method to be considered here is one commonly used in practice for allocating the costs of multipurpose water development projects. The SCRB method is based on the simple and appealing idea that joint costs should be allocated, more or less, in proportion to the willingness of the user to pay. The 'more or less' element is introduced because the proportional allocation is only performed after first assigning to each user his marginal (or separable) cost and then taking as the criterion of proportionality each user's willingness to pay minus the marginal cost already allocated.

TABLE 3. The Shapley Values for Municipalities A, B, and C

\begin{tabular}{lccc}
\hline & \multicolumn{3}{c}{ Marginal Contribution, dollars $\times 10^{6}$} \\
\cline { 2 - 4 } Order of Signing & $\mathbf{A}$ & $\mathrm{B}$ & $\mathrm{C}$ \\
\hline ABC & 6.5 & 3.8 & 0.3 \\
ACB & 6.5 & 2.6 & 1.5 \\
BAC & 6.1 & 4.2 & 0.3 \\
BCA & 5.3 & 4.2 & 1.1 \\
CAB & 6.5 & 2.6 & 1.5 \\
CBA & 5.3 & 3.8 & 1.5 \\
Total & 36.2 & 21.2 & 6.2 \\
Shapley value* & 6.033 & 3.533 & 1.033 \\
\hline
\end{tabular}

*The Shapley value is calculated by dividing the sum of the possible marginal contributions for each municipality (Total) by the number of possible orders of signing (6). 
Specifically, let $b(i)$ be the benefit to user $i$ and $c(i)$ his alternative cost. Evidently, $i$ would not be willing to pay more than $\min \{b(i), c(i)\}$ (sometimes called his justifiable expenditure) to participate in the joint project. The marginal cost of including $i$ is $c^{\prime}(i)=c(N)-c(N-i)$, which is less than or equal to $c(i)$ because the cost function is subadditive. In the SCRB nomenclature, $i$ 's 'remaining benefit' $r(i)$ is his willingness to pay minus his marginal cost: $r(i)=\{\min b(i)$, $c(i)\}-c^{\prime}(i)$. Note that if $r(i)<0$, then $c^{\prime}(i)>b(i)$ and user $i$ should not be included in the project. Therefore we can assume that all remaining benefits $r(i)$ are nonnegative. The remaining costs are $c(N)-\Sigma_{N} c^{\prime}(j)$ and are allocated in proportion to the remaining benefits:

$$
v_{i}=c^{\prime}(i)+\left[r(i) / \sum_{N} r_{j}\right]\left[c(N)-\sum_{N} c^{\prime}(i)\right]
$$

Using the marginal costs given in section 3 , the remaining benefits (in millions of dollars) for $\mathrm{A}, \mathrm{B}$, and $\mathrm{C}$ are calculated to be

$$
\begin{array}{r}
r(A)=6.5-5.3=1.2 \\
r(B)=4.2-2.6=1.6 \\
r(C)=1.5-0.3=1.2 \\
\text { Total }=4.0
\end{array}
$$

The nonseparable costs are $10.6-(5.3+2.6+0.3)=2.4$, so the SCRB allocation (in millions of dollars) is:

$$
\begin{aligned}
& y_{A}=5.3+\frac{1.2}{4.0}(2.4)=6.02 \\
& y_{B}=2.6+\frac{1.6}{4.0}(2.4)=3.56 \\
& y_{C}=0.3+\frac{1.2}{4.0}(2.4)=1.02
\end{aligned}
$$

Variations of the SCRB method include proposals for allocating the nonseparable costs in proportion to some criterion, such as use, priority of use, or population [James and Lee, 1971]. The difficulty with the SCRB method is that the simple underlying idea of allocating costs in proportion to benefits is lost by the ad hoc introduction of marginal costs; this leads to some strange results, as will be shown in section 8 .

\section{A Case Study of Sweden}

The area studied consists of 18 municipalities in the Skåne region of southern Sweden (Figure 2). At present, most of the municipal water supply is drawn from three sources: local groundwater, and two separate pipeline systems which distribute water from two lakes, Vombsjön and Ringsjön.

As early as the 1940's certain municipalities in the area realized that local water sources might not be sufficient to meet future demand and turned their attention to off-site sources. An association called the Sydvatten Company was formed by several of them to plan the long-term water supply for the region. In the late 1960's this group started to design a major project for obtaining water from a lake outside the region (Lake Bolmen) via an 80-km tunnel.

The viability of the project depends on the number of additional municipalities that can be induced to participate in the project. This in turn depends on how much these municipalities will be obliged to pay for participation, bearing in mind the availability and costs of developing their own on-site resources. Moreover, there have been several unforeseen developments since the initiation of the project (e.g., greatly escalated costs, more optimistic estimates of local resources, and lower rates of demand growth), and these have brought the present population-based cost allocation procedure into question.

The basic concepts and methods developed in the preceding sections can be illustrated by applying them to the Swedish case over the decade 1970-1980, since data and forecasts for this period are readily available. The Sydvatten tunnel project mentioned earlier is not expected to have any impact on water supply until the late 1980's, and so only conventional alternatives can be considered for meeting incremental demands over the period studied (e.g., extending the capacity of the pipeline system and increasing the use of local groundwater sources where feasible). The base year was taken to be 1970 , and a water supply system was designed to satisfy the municipal 'requirements' for 1980 as they were forecast in 1970 . The different cost allocation methods described above were then applied to the system to examine the relative positions of the different municipalities. The results help clarify the way in which the different methods work, and why some of them may be preferable to others in practice.

\subsection{Identifying Independent Actors}

The first problem in defining the cost function is to identify the independent actors in the system. To develop the costs for each of the $2^{18}-1=262,143$ possible groupings of the 18 municipalities would be impractical and unrealistic. In practice the municipalities fall into natural groups based on past associations, geographical location, existing water transmission systems, and hydrological and geographical features determining the natural routes for water transmission networks.

A careful study of these conditions led to the grouping of the 18 municipalities into six independent units as shown in Figure 2. Group H, for example, consists of those municipalities which were already connected by the Ringsjön water supply system in 1970, together with the municipality of Svalöv, which would be located in the middle of the main transmission route. These groups are treated as single actors in the following analysis of alternative costs. Once a cost allocation for the groups has been determined, a further allocation of costs among the municipalities within each group could be made using a similar approach; however, this may not give the same result as if all municipalities were treated separately. The 1970 populations and forecast incremental water demands of each group are shown in Table 4.

\subsection{Ambiguities in Defining the Cost Function: Direct Costs}

In practice, ambiguities in defining the cost function arise due to the problem of direct costs, that is, costs that would be incurred by a given municipality no matter what course of action is pursued. For example, local water distribution systems are required within municipalities whether the water is supplied jointly or separately. The cost associated with municipal distribution systems may therefore be regarded as 
TABLE 4. Populations and Incremental Water Demands of the Six Municipal Groups

\begin{tabular}{lcc}
\hline Group & $\begin{array}{c}\text { Population, } \\
\times 10^{3}\end{array}$ & $\begin{array}{c}\text { Incremental } \\
\text { Water Demand, } \\
\times 10^{6} \mathrm{~m}^{3} / \mathrm{yr}\end{array}$ \\
\hline A & 85.0 & 6.72 \\
H & 176.3 & 8.23 \\
K & 26.8 & 3.75 \\
L & 69.0 & 3.53 \\
M & 287.3 & 14.64 \\
T & 59.5 & 6.21 \\
Total & 703.9 & 43.08 \\
\hline
\end{tabular}

a direct cost. In principle these costs could be excluded from the cost function on the grounds that they can be allocated independently. However, in practice the borderline between direct and indirect costs is not always clear. In some municipalities, for instance, the water delivered by the regional supply network must first be pumped up to a reservoir before distribution within the municipality; the facilities required for pumping depend on the pressure at the end of the transmission network. Thus the costs of these distribution facilities may not be independent of the method by which the water is supplied. The definition of the cost function naturally depends on the fraction of these costs treated as direct costs.

Since the definition of the cost function is always somewhat arbitrary in practice, it is desirable to choose a cost allocation method that is insensitive to the inclusion of direct costs. One of the difficulties of the SCRB method is that in some cases it is sensitive to the inclusion of direct costs. None of the game theory methods considered here suffers from this drawback.

\subsection{Calculating the Cost Function}

The water supply system includes two lakes (Vombsjön and Ringsjön), one major groundwater aquifer (Alnarp), and other minor on-site sources. The possible routes of a water transmission network (based on a preliminary analysis) are shown in Figure 2. To avoid inconsistencies in defining the cost function, it was assumed for the purpose of this study that the pressure at each demand point does not depend on the method by which the water is transmitted to that point. Thus the cost of distributing the water within each municipality does not depend on the arrangement by which the water is supplied, so this element can be treated as a direct cost and excluded from the cost function. The water delivered to each municipality was assumed to be of the same quality. The water was treated to bring it up to this level at the source, and the cost of treatment was included in the cost function.

Cost functions for transmitting and treating water were computed using standard formulas (see Appendix A), and the total costs $c(S)$ associated with the least cost combination of alternative supply sources were estimated for each coalition $S$. The results are shown in Table 5; commas signify that the least cost option of that coalition is to break up into the subcoalitions indicated. The unit costs (per
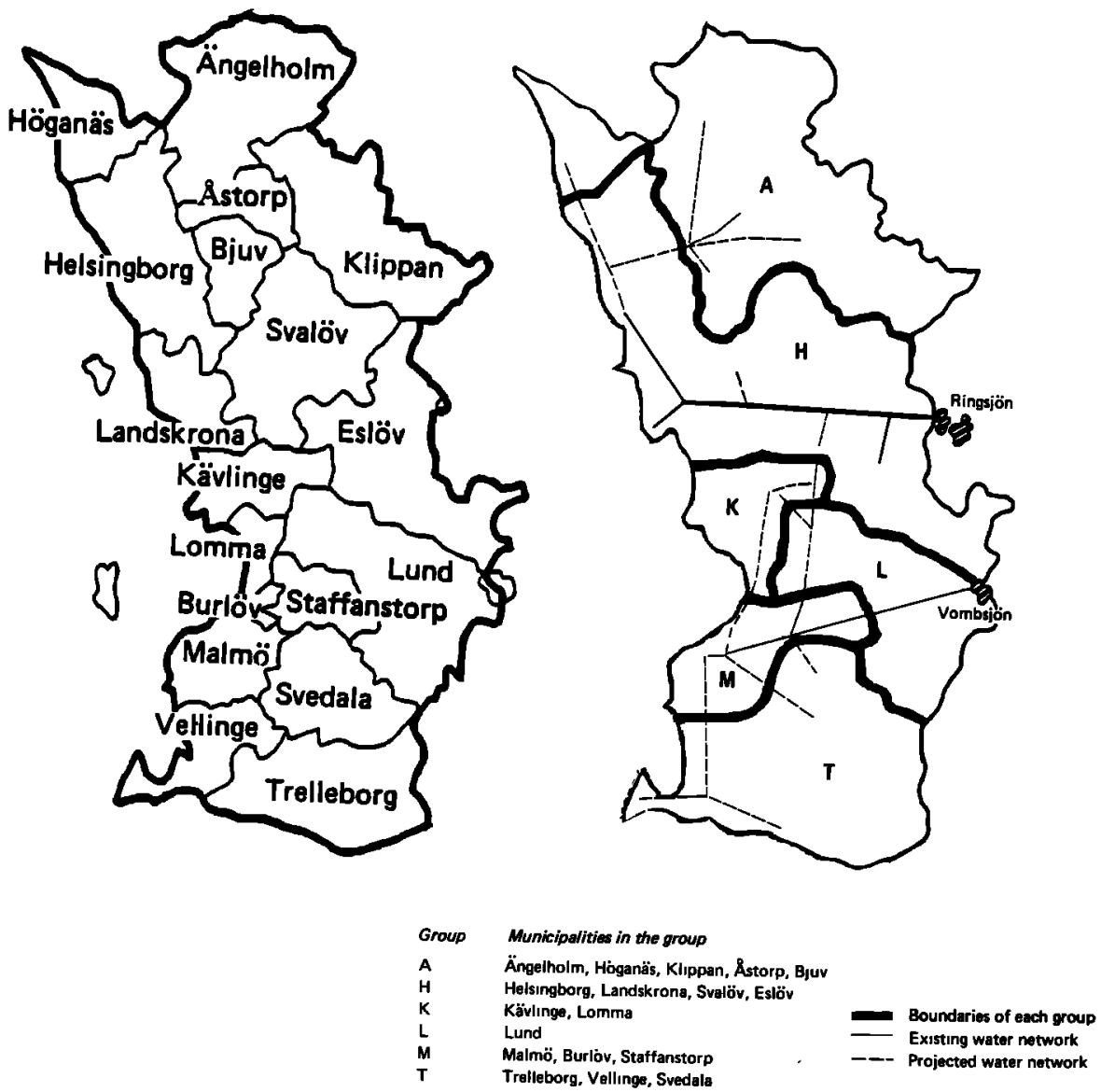

Fig. 2. The region studied in Skone, Sweden, and its divisions into groups of municipalities. 
TABLE 5. Total Costs for Various Joint Supply Systems

\begin{tabular}{lclclc}
\hline Group & Total Cost & Group & Total Cost & Group & Total Cost \\
\hline A & 21.95 & AHK & 40.74 & AHKL & 48.95 \\
H & 17.08 & AHL & 43.22 & AHKM & 60.25 \\
K & 10.91 & AH, M & 55.50 & AHK, T & 62.72 \\
L & 15.88 & AH, T & 56.67 & AHL, M & 64.03 \\
M & 20.81 & A, K, L & 48.74 & AHL, T & 65.20 \\
T & 21.98 & A, KM & 53.40 & AH, MT & 74.10 \\
& & A, K, T & 54.84 & A, K, LM & 63.96 \\
AH & 34.69 & A, LM & 53.05 & A, K, L, T & 70.72 \\
A, K & 32.86 & A, L, T & 59.81 & A, K, MT & 72.27 \\
A, L & 37.83 & A, MT & 61.36 & A, LMT & 73.41 \\
A, M & 42.76 & HKL & 27.26 & HKL, M & 48.07 \\
A, T & 43.93 & HKM & 42.55 & HKL, T & 49.24 \\
HK & 22.96 & HK, T & 44.94 & HKMT & 59.35 \\
HL & 25.00 & HL, M & 45.81 & HLMT & 64.41 \\
H, M & 37.89 & HL, T & 46.98 & KLMT & 56.61 \\
H, T & 39.06 & H, MT & 56.49 & AHKL, T & 70.93 \\
K, L & 26.79 & K, LM & 42.01 & AHKLM & 69.76 \\
KM & 31.45 & K, L, T & 48.77 & AHKMT & 77.42 \\
K, T & 32.89 & K, MT & 50.32 & AHLMT & 83.00 \\
LM & 31.10 & LMT & 51.46 & AKLMT & 73.97 \\
L, T & 37.86 & & & HKLMT & 66.46 \\
MT & 39.41 & & & AHKLMT & 83.82 \\
\hline
\end{tabular}

Values in Skr $\times 10^{6}$

million $\mathrm{m}^{3} \mathrm{yr}^{-1}$ ) are $\mathrm{A}, 3.27 ; \mathrm{H}, 2.08 ; \mathrm{K}, 2.91 ; \mathrm{L}, 4.50 ; \mathrm{M}$, 1.42; $\mathrm{T}, 3.51$.

The cost function reveals the relative strength of the different actors, which depends on factors such as the cost and availability of local resources and access to the resources of others. For example, $L$ finds that the unit cost of going alone is high; though it is located close to the major regional sources (Ringsjön and Vombsjön), it owns neither. Hence it has a strong incentive to participate in a joint scheme with the owners of the two sources, $\mathrm{H}$ and $\mathrm{M}$. $\mathrm{H}$ and $M$ have the lowest unit costs because they own the two systems, but they can reduce their unit costs even further by including other municipalities in a joint scheme, due to economies of scale. However, the system owned by $\mathbf{H}$ (Ringsjön) has a greater excess capacity than that owned by M (Vombsjön). Hence the incremental cost of other municipalities joining in a coalition with $\mathrm{M}$ is higher than the incremental cost of joining with $\mathrm{H}$. The effect is that $\mathrm{H}$ has more to offer its partners in a coalition than does $\mathrm{M}$, and this will be reflected in the final cost allocation.

\section{Comparison of Methods}

Using the cost function developed above, we now compare the cost allocations of the different methods described in the preceding sections. We assume that all groups participate in a joint scheme at a total cost of Skr 83.82 million (Skr $=$ Swedish kronas). The results obtained from the two proportional allocation schemes based on demand and population and from the SCRB method are shown in Table 6, as are the Shapley value, the nucleous, the weak nucleolus, and the proportional nucleolus. The cost shares allocated to each group by the seven methods are shown in Table 7 for ease of comparison. Note that the 'proportional' allocations differ markedly from the others. A comparison of the proportional allocations with the costs of going alone reveals that these methods would charge some participants in the joint project more than they would have had to pay on their own. Allocation by demand penalizes $M$ for participating, while allocation by population penalizes both $H$ and $M$.

This failure to satisfy the requirement of individual rationality is based on the fact that proportional methods do not take into account crucial differences among the participants in their access to sources of supply. The proportional procedures work against $H$ and $M$, which have large populations, and favor the outlying regions $A$ and $T$. The inclusion of $A$ and $T$ is relatively costly, despite their smaller populations, because they are both remote from the major sources of supply. This fact is reflected in their high marginal costs

TABLE 6. Cost Allocations of Skr 83.82 Million by Seven Methods

\begin{tabular}{|c|c|c|c|c|c|c|c|}
\hline \multirow[b]{2}{*}{ Allocation Method } & \multicolumn{6}{|c|}{ Cost Allocations, $\mathrm{Skr} \times 10^{6}$} & \multirow[b]{2}{*}{ Total } \\
\hline & $\mathbf{A}$ & $\mathbf{H}$ & $\mathbf{K}$ & $\mathrm{L}$ & $\mathbf{M}$ & $\mathbf{T}$ & \\
\hline $\begin{array}{l}\text { Proportional to population } \\
\text { Proportional to demand } \\
\text { SCRB } \\
\text { Shapley value } \\
\text { Nucleolus } \\
\text { Weak nucleolus } \\
\text { Proportional nucleolus }\end{array}$ & $\begin{array}{l}10.13 \\
13.07 \\
19.54 \\
20.01 \\
20.35 \\
20.03 \\
20.36\end{array}$ & $\begin{array}{l}21.00 \\
16.01 \\
13.28 \\
10.71 \\
12.06 \\
12.52 \\
12.46\end{array}$ & $\begin{array}{l}3.19 \\
7.30 \\
5.62 \\
6.61 \\
5.00 \\
3.94 \\
3.52\end{array}$ & $\begin{array}{r}8.22 \\
6.87 \\
10.90 \\
10.37 \\
8.61 \\
9.07 \\
8.67\end{array}$ & $\begin{array}{l}34.22 \\
28.48 \\
16.66 \\
16.94 \\
18.32 \\
18.54 \\
18.82\end{array}$ & $\begin{array}{r}7.07 \\
12.08 \\
17.82 \\
19.18 \\
19.49 \\
19.71 \\
19.99\end{array}$ & $\begin{array}{l}83.82 \\
83.82 \\
83.82 \\
83.82 \\
83.82 \\
83.82 \\
83.82\end{array}$ \\
\hline Individual alternative costs & 21.95 & 17.08 & 10.91 & 15.88 & 20.81 & 21.98 & 108.61 \\
\hline Marginal costs & 17.36 & 9.85 & 0.82 & 6.40 & 12.89 & 14.06 & 61.38 \\
\hline
\end{tabular}


(Table 6). Though A and T should be charged at least the marginal cost of including them, both proportional methods fail to do so. This is one serious disadvantage of the proportional allocation methods.

Seemingly more reasonable than the proportional schemes, but actually almost as ill behaved, is the SCRB method. This method is individually rational only if the alternative costs are less than the corresponding benefits (as assumed in the present case); otherwise this may not be so. It also may not satisfy group rationality. For example, the three adjacent municipal groups $H, K$, and $L$ can provide municipal water for themselves at a cost of Skr 27.26 million, but the SCRB method would assess them a total of Skr 29.80 million if they all shared in a regional facility. It would not be in the interest of $\mathrm{H}, \mathrm{K}$, and $\mathrm{L}$ to participate on the basis of such an assessment because they are then, in effect, being forced to subsidize the other participants. Since there exist assessments in which no coalition subsidizes any other, such an allocation could be considered inequitable.

That the SCRB method suffers from this defect is not surprising, since it considers only the marginal costs of including individual participants, not the marginal costs of coalitions. In this case the marginal cost of including both $\mathbf{M}$ and $T$ is much higher than the sum of their individual marginal costs, since if one is already being served, the additional expense of serving the other (or individual marginal cost) is low. Thus while it is not necessary to estimate all cost elements in order to calculate the SCRB allocation, this shortcut has a price: it may result in a less equitable final distribution.

By contrast, a calculation of the Shapley value requires the alternative costs for all possible subsets. Unfortunately, however, this allocation also fails the group rationality and marginality tests. In particular, the Shapley value assesses the coalition HKL at $\mathbf{S k r} \mathbf{4 3 0 , 0 0 0}$ more than its alternative cost.

Since group rationality and marginal cost coverage seem to be essential from the standpoint of equity, that is, to provide sufficient incentives for cooperation, the remaining three methods, the nucleolus, weak nucleolus, and proportional nucleolus, are potentially more desirable than those discussed above, as they always produce a core imputation if one exists. Is there any basis for preferring one method to another?

A general point raised in the early study by Ransmeier [1942] is that a method should be able to adapt to changing conditions. The need for adaptability is evident in the fact that, typically, total project costs are not known precisely until after the project has been completed. Since the cost allocation method is usually agreed upon before the project has been started, it must specify how different levels of total costs should be allocated. Such an agreement will initially be based on some best estimate of the costs of alternatives, but once an agreement has been reached, the alternatives must be abandoned. It is therefore sufficient that a method specify how different levels of total costs should be allocated for fixed levels of alternative costs.

An elementary requirement of any such method is that if total costs increase then no participant will be charged less; conversely, if total costs decrease, no participant will be required to pay more. This property is called monotonicity [Megiddo, 1974] and is fundamental in problems of fair division [see, for example, Huntington, 1928; Balinski and Young, 1982].
Several of the methods considered so far do not possess this fundamental property. As an example, suppose that the total cost of the Swedish project involved an overrun of Skr 4 million, bringing the total to Skr 87.82 million (see Table 7). This situation could arise if the method used for supplying the whole coalition involved components that would not be cost-effective in any smaller coalition; for example, the large fixed costs required for an interbasin transfer project. In fact a project of this nature is currently under construction in Sweden, and the costs are already substantially more than predicted.

Comparing the new allocations with the old (Table 6), we notice that the nucleolus requires $\mathrm{K}$ to pay less even though the total cost of the project has increased. Similarly, the SCRB method allocates K Skr 160,000 less when costs increase. This nonmonotonic behavior suggests that the nucleolus and SCRB methods may not be reasonable cost allocation procedures. (The nonmonotonicity of the nucleolus was first pointed out by Megiddo [1974]).

On the other hand, it is clear that methods which allocate costs in proportion to some single criterion, like population or use, must be monotonic. This is also the case for the Shapley value, the weak nucleolus, and the proportional nucleolus. For the Shapley value and weak nucleolus, any change in total costs is distributed equally among the actors (see Appendix B). However, this particular way of dividing changes in costs is questionable: Why should all participants have to share unforeseen joint expenses equally, when their shares in the enterprise are very different? Perhaps a fairer way of measuring the participants' shares in the enterprise is not by their costs (most of which they might well have incurred acting alone), but rather by the amounts that they save by virtue of taking part in the enterprise as opposed to going alone. A reasonable scheme would be to divide any unforeseen costs in proportion to the benefits enjoyed. This is precisely the way in which the proportional nucleolus works (see Appendix B).

\section{SUMMARY}

Given the practical need to allocate the costs of a joint water resource facility among the different users, it is necessary to choose rationally among the many different methods of allocation available. Using a definite computational procedure itself provides some semblance of rationality. However, the justification of a method does not lie in the computational procedure employed, but in its behavior in practice. Thus it is necessary to compare the different methods on the basis of principle.

Among the most important of these principles are individual rationality, group rationality (equivalently marginal cost coverage), insensitivity to the definition of direct costs, monotonicity in costs, and no payments to dummies. A more general principle is simplicity in terms of computational and informational demands. Seven different methods (including two proportional methods) have been selected for comparison from among those discussed in the project evaluation and game theory literature and their performance evaluated by application to an actual municipal cost allocation problem in Sweden. The properties of the methods in general problems are summarized in Table 8, which shows the principles violated by each.

The game theory methods all suffer from the disadvantage that they are fairly complicated and require detailed information on costs. All of the methods except for the simplest 
TABLE 7. Allocations With a Cost Overrun of Skr 4.0 Million

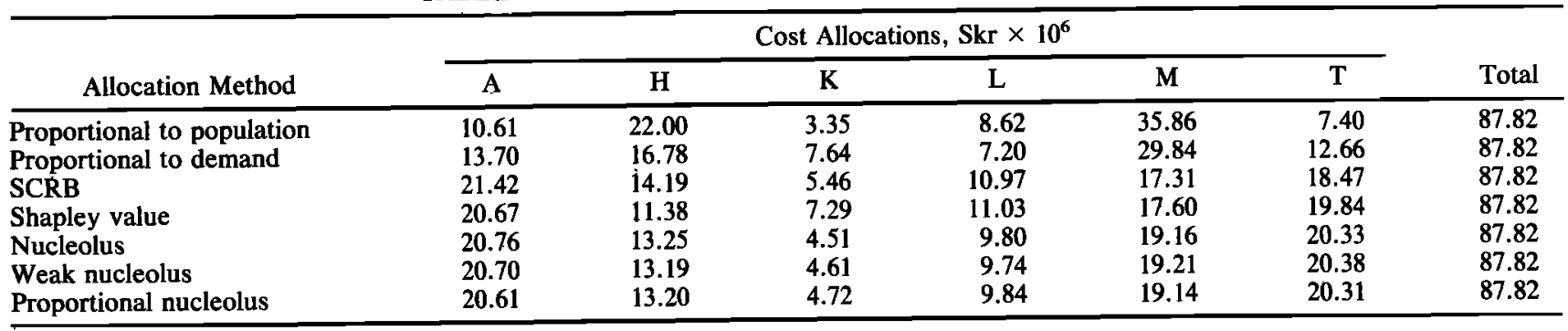

proportional allocation schemes rely on information about demands and the optimal scale of development that in practice may be unreliable or nonexistent. This is particularly true for long-term investment projects such as the Swedish case discussed above. It is therefore not surprising to find that a simple scheme based on allocating costs in proportion to the population was the one actually chosen.

\section{Appendix A: Cost of Water Supply Network}

This section describes the procedure used to determine the capital costs of pipes and pumps necessary to supply the required amounts of water to the demand points shown in Table Al. The distances between points (in parentheses) and their elevations are shown in Figure A1. It is assumed that the pressure at each demand point does not depend on the arrangement by which the water is supplied. This allows us to treat each arc of the transmission network independently. The cost analysis of the network is therefore carried out arc by arc.

The cost of water transmission includes the following components:

Cost of pipelines

$$
C_{1}=c_{1} L=\left(\gamma+\alpha D^{\beta}\right) L \quad \mathrm{Skr}
$$

Cost of pumps

$$
C_{2}=c_{2} f P \quad \mathrm{Skr}
$$

Cost of electricity

$$
C_{3}=c_{3} P \quad \mathrm{Skr} / \mathrm{yr}
$$

where

$c_{1} \quad$ unit cost of piping, $\mathrm{Skr} / \mathrm{m}$;

$L \quad$ length of pipe, $\mathrm{m}$;

$c_{2}$ unit cost of pump, $\mathrm{Skr} / \mathrm{kW}$;

$f$ safety factor;

$c_{3} \quad$ unit cost of electricity, $\mathrm{Skr} / \mathrm{kW} \mathrm{yr}$;

$P$ effective capacity of pump, equal to $(9.81 / E) Q H$; $\mathbf{k W}$;
$Q$ flow of water in pipe, $\mathrm{m}^{3} / \mathrm{s}$;

$H$ required pumping head, equal to $H_{0}+I L ; \mathrm{m}$;

$H_{0}$ difference in altitude between origin and destination of pipe, $\mathrm{m}$;

I hydraulic gradient;

$E$ pumping efficiency;

$D$ pipe diameter, $\mathrm{m}$;

$\alpha, \beta, \gamma$ coefficients.

The total annual cost of transmission is given by

$$
C=\left(C_{1}+C_{2}\right) C R F+C_{3}
$$

where

$$
C R F=\frac{i(1+i)^{n}}{(1+i)^{n}-1}=\text { capital recovery factor }
$$

$i$ is the interest rate, and $n$ is the amortization period in years. The total cost $C$ is a function of the pipe diameter $D$, the flow $Q$, the pumping head $H$, and the length of pipe $L$. These factors are related by the Hazen-Williams formula:

$$
H=10.7(C w)^{-1.85} D^{-4.87} Q^{1.85} L
$$

where $C w$ is the Hazen-Williams coefficient.

The economical pipe diameter $D^{*}$ is obtained as a function of the flow $Q$ by letting $\partial C / \partial D=0$ :

$$
D^{*}=\left(\frac{4.87 a b}{\alpha \beta C R F}\right)^{1 /(\beta+4.87)} Q^{2.85 /(\beta+4.87)}
$$

where

$$
a=\left(c_{2} f C R F+c_{3}\right)(9.81 / E) \quad b=10.7(C w)^{-1.85}
$$

\begin{tabular}{|c|c|c|c|c|c|c|}
\hline & Proportional & SCRB & Shapley & Nucleous & $\begin{array}{c}\text { Weak } \\
\text { Nucleus }\end{array}$ & $\begin{array}{l}\text { Proportional } \\
\text { Nucleus }\end{array}$ \\
\hline Individual rationality & $X$ & $\mathrm{X}$ & & & $\mathbf{X}$ & \\
\hline Group rationality & $\mathrm{X}$ & $\mathrm{X}$ & $X$ & & & \\
\hline Direct costs & $\mathbf{x}$ & $\mathbf{X}$ & & & & \\
\hline Monotonicity & & $\mathbf{X}$ & & $\mathbf{X}$ & & \\
\hline $\begin{array}{l}\text { Dummy } \\
\text { Simplicity }\end{array}$ & $X$ & $\mathbf{X}$ & $\mathrm{X}$ & $\mathbf{X}$ & $\begin{array}{l}\mathbf{X} \\
\mathbf{X}\end{array}$ & $\mathbf{x}$ \\
\hline
\end{tabular}

Similarly, the economical hydraulic gradient $I^{*}$ is obtained as

$$
I^{*}=\left(\frac{\alpha \beta C R F}{4.87 a}\right)^{\beta / 4.87} Q^{(1.85 \beta-4.87) /(\beta+4.87)}
$$

The parameters are determined from the Swedish data as follows: $\alpha=477 \mathrm{Skr} ; \beta=1.60 ; \gamma=150 \mathrm{Skr} ; E=0.63 ; C w=$

TABLE 8. Principles Violated by Each Method

$\mathrm{X}$ denotes that the principle has been violated by that method. 
TABLE A1. Incremental Water Demand for all Municipalities in the Study Area for the Period 1970-1980

\begin{tabular}{lclc}
\hline Municipality & $\begin{array}{c}\text { Incremental } \\
\text { Water Demand, } \\
\times 10^{6} \mathrm{~m}^{3} / \mathrm{yr}\end{array}$ & Municipality & $\begin{array}{c}\text { Incremental } \\
\text { Water Demand, } \\
\times 10^{6} \mathrm{~m}^{3} / \mathrm{yr}\end{array}$ \\
\hline Ängelholm & 2.65 & Lund & 3.53 \\
Höganäs & 1.48 & Malmö & 10.66 \\
Klippan & 0.60 & Burlöv & 1.67 \\
Åstorp & 0.63 & Staffanstorp & 2.30 \\
Bjuv & 1.36 & Trelleborg & 1.26 \\
Helsinghnro & $1 \mathrm{\kappa n}$ & Vellinge* & 2.30 \\
Landskrona & 1.80 & Räng* & 0.57 \\
Svalöv & 0.50 & Skanör* & 0.25 \\
Eslöv & 1.32 & Svedala* & 1.10 \\
Kävlinge & 2.74 & Bara* & 0.73 \\
Lomma & 1.01 & & \\
\hline
\end{tabular}

Taken from Sydvatten, Prognos 73.

"Bara is included in the municipality of Svedala; Vellinge, Räng, and Skanör constitute a single municipality.

$100 ; f=1.33 ; C R F=0.0871$, based on $i=0.06, n=20$ years; $c_{2}=1893 \mathrm{Skr} / \mathrm{kW} ; c_{3}=613 \mathrm{Skr} / \mathrm{kW}$ yr. The results are $D^{*}=0.928 Q^{0.43}, I^{*}=2.99 Q^{-0.28} \times 10^{3}, C_{1}=(150+$ $\left.477 \mathrm{D}^{1.60}\right) \mathrm{L}, C_{2}=39.2 \mathrm{QH} \times 10^{3}$, and $C_{3}=9.54 \mathrm{QH} \times 10^{3}$.

The cost of treating water at Vombsjön includes the costs of filtration, pumping, and chlorination. The treatment at Ringsjön consists of screening, sedimentation, coagulation, and filtration. Unfortunately, the particular cost data on these unit processes were not available, so capital costs and the costs of operation and maintenance $(\mathrm{O} / \mathrm{M})$ were estimated using available data. The cost of treating on-site groundwater includes the costs of pumping, filtration, and chlorination, and these were also estimated. The results of the cost analysis are given in Table A2, where $\tilde{Q}$ is the flow of water through the treatment plant in millions of cubic meters per year.

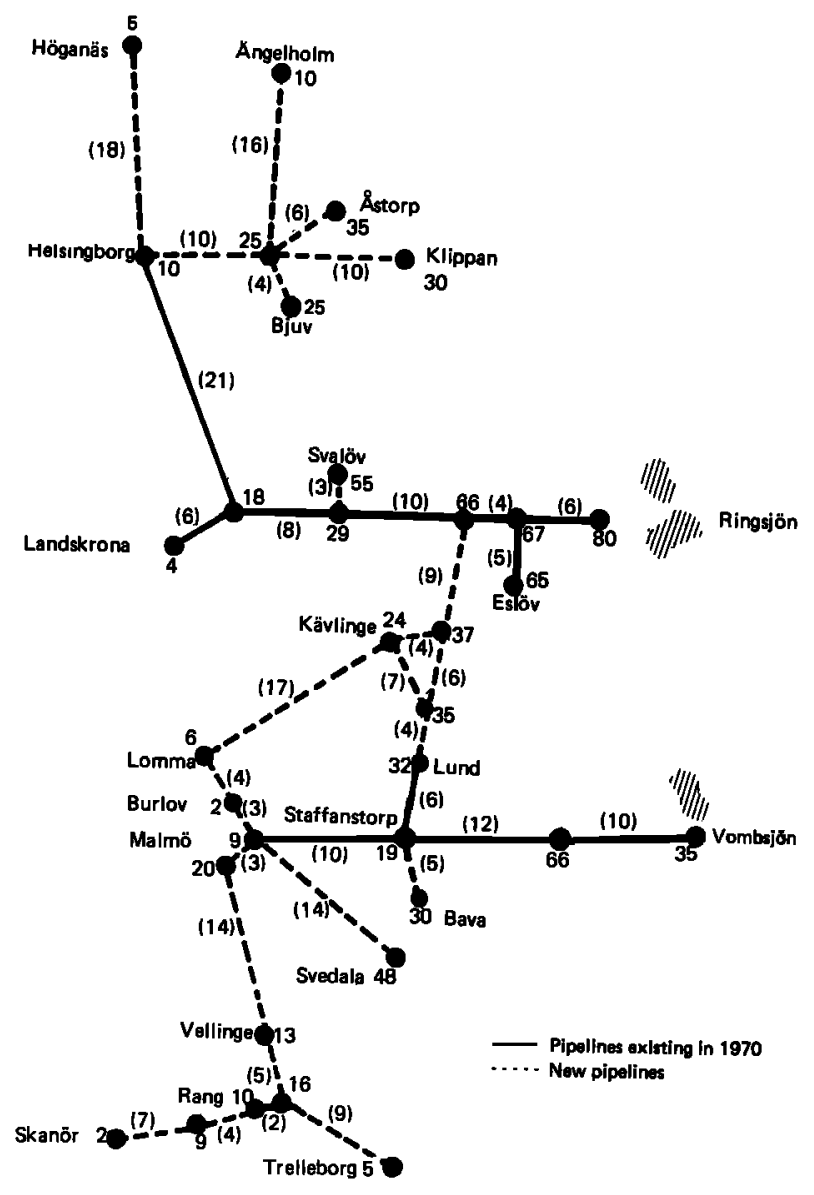

Fig. A1. The water transmission network. The number at each node represents the elevation in meters; the number in parentheses on each arc represents the distance in kilometers. 
TABLE A2. The Cost of Providing Water From the Three Sources

\begin{tabular}{llll}
\hline Water Source & \multicolumn{1}{c}{ Cost } & \multicolumn{1}{c}{ Value } & Unit, $\times 10^{6}$ \\
\hline Vombsjon & Capital & $C_{K}{ }^{V}=2.31 \tilde{Q}^{0.74}$ & Skr \\
& O/M & $C_{M}=0.162 \bar{Q}^{0.91}$ & Skr/yr \\
Ringsjön & Capital & $C_{K}{ }^{R}=3.68 \bar{Q}^{0.64}$ & Skr \\
& O/M & $C_{M}=0.410 \tilde{Q}^{0.64}$ & Skr/yr \\
Groundwater & Capital & $C_{K}^{G}=2.38 \bar{Q}^{8.58}$ & Skr \\
& $\mathrm{O} / \mathrm{M}$ & $C_{M}{ }^{G}=0.263 \bar{Q}^{0.82}$ & Skr/yr \\
\hline
\end{tabular}

\section{APPENDIX B: MONOTONICITY}

The weak least core is the set of optimal solutions to the linear program

$\min \varepsilon$

subject to

$$
\begin{gathered}
\sum_{N} x_{i}=v(N) \\
\sum_{S} x_{i} \geq \nu(S)-\varepsilon|S| \quad \forall S \subset N
\end{gathered}
$$

which is equivalent to

$$
\min \sum_{N}\left(x_{i}+\varepsilon\right)
$$

subject to

$$
\begin{gathered}
\sum_{N} x_{i}=v(N) \\
\sum_{S}\left(x_{i}+\varepsilon\right) \geq v(S) \quad \forall S \subset N
\end{gathered}
$$

This is equivalent to solving

$$
\min \sum x_{i}^{\prime}
$$

subject to

$$
\sum_{s} x_{i}^{\prime} \geq v(S) \quad \forall S \subset N
$$

and letting $x_{i}=x_{i}{ }^{\prime}-\varepsilon$, where $\varepsilon=\left\{\Sigma_{N} x_{i}{ }^{\prime}-\nu(N)\right\} / n$. Since the $x^{\prime}$ program is independent of $v(N)$, an increase in $v(N)$ simply has the effect of increasing $\varepsilon$-and hence every component $x_{i}$-by the same amount. The same holds for the weak nucleolus; hence the weak nucleolus is monotonic. The proof of monotonicity of the Shapley value is left to the reader.

The proportional least core is the set of optimal solutions to the linear program

\section{$\min t$}

subject to

$$
\begin{gathered}
\sum_{N} x_{i}=v(N) \\
\sum_{S} x_{i} \geq(1-t) \nu(S) \quad \forall S \subset N
\end{gathered}
$$

where we assume that $v(S)>0$ for some $S \neq N$ to ensure existence. This is equivalent to solving (12) and letting $x_{i}=$ $x_{i}^{\prime} v(N) / \Sigma_{N} x_{i}^{\prime}$. An increase in $v(N)$ by a common proportion simply has the effect of increasing every component of $x_{i}$ by the same proportion. The same holds for the proportional nucleolus; hence it is monotonic.

Acknowledgments. The authors gratefully acknowledge the help of Lennart de Mare of the University of Lund in preparing this study, the comments of $O$. Menshikova and $I$. Menshikov of the Computing Center of the Academy of Sciences of the U.S.S.R., and the advice and encouragement of Janusz Kindler of the International Institute of Applied Systems Analysis, where this work was accomplished.

\section{REFERENCES}

Balinski, M. L., and H. P. Young, Fair Representation, Yale University Press, New Haven, Connecticut, 1982.

Baumol, W., and D. Bradford, Optimal departures from marginal cost pricing, Am. Econ. Rev., 60, 265-283, 1970.

Bogardi, I., and F. Szidarovsky, Application of game theory in water management, Appl. Math. Model., 1, 16-20, 1976.

Charnes, A., J. Rousseau, and L. Seiford, Complements, mollifiers, and the propensity to disrupt, Int. J. Game Theory, 7, 37-50, 1978.

Eckstein, O., Water Resource Development, Harvard University Press, Cambridge, Massachusetts, 1958.

Faulhaber, G., Cross-subsidization: Pricing in public enterprises, Am. Econ. Rev., 65, 966-977, 1975.

Federal Inter-Agency River Basin Committee, Proposed practices for economic analysis of river basin projects, report, Washington, D.C., 1950.

Grotte, J. H., Computation of and observations on the nucleolus, and the central games, M.Sc. thesis, Cornell Univ., Ithaca, N.Y., 1970 .

Grotte, J. H., Dynamics of cooperative games, Int. J. Game Theory, $5,27-64,1976$.

Heaney, J. P., Efficiency/equity analysis of environmental problems: A game theoretic perspective, in Applied Game Theory, edited by S. J. Brams, A. Schotter, and G. Schwodiauer, pp. 352369; Physica-Verlag, Vienna, 1979.

Huntington, E. V., The apportionment of representatives in Congress, Trans. Am. Math. Soc., 30, 85-110, 1928.

Inter-Agency Committee on Water Resources, Proposed practices for economic analysis of river basin projects, report, Washington, D.C., 1958.

James, L. D., and R. R. Lee, Economics of Water Resources Planning, McGraw-Hill, New York, 1971.

Loehman, E., and A. Whinston, An axiomatic approach to cost allocation for public investment, Public Finance $Q$., 1, 236-251, 1974.

Loehman, E., J. Orlando, J. Tschirhart, and A. Whinston, Cost allocation for a regional wastewater treatment system, Water Resour. Res., 15, 193-202, 1979.

Loughlin, J. C., The efficiency and equity of cost allocation methods for multipurpose water projects, Water Resour. Res., 13, 8-14, 1977.

Maschler, M., B. Peleg, and L. S. Shapley, Geometric properties of the kernel, nucleolus, and related solution concepts, Math. Oper. Res., 4, 303-338, 1979.

Megiddo, N., On the nonmonotonicity of the bargaining set, the kernel, and the nucleolus of a game, SIAM J. Appl. Math., 27, 355-358, 1974.

Okada, N., A game-theoretic approach to the analysis of area-wide, multi-modal water utilization system, Tottori Daigaku Kogakubu Kenkyu Hokoku, 8, 1977.

Ramsey, F., A contribution to the theory of taxation, Econ. J., 37, 47-61, 1927.

Ransmeier, J. S., The Tennessee Valley Authority: A Case Study in the Economics of Multiple Purpose Stream Planning, Vanderbilt University Press, Nashville, Tennessee, 1942.

Schmeidler, D., The nucleolus of a characteristic function garne, SIAM J. Appl. Math., 17, 1163-1170, 1969.

Shapley, L. S., A value for n-person games, in Contributions to the Theory of Games, II, Annals Math. Stud. 28, edited by H. W. Kuhn and A. W. Tucker, pp. 303-306, Princeton University Press, Princeton, New Jersey, 1953.

Shapley, L. S., and M. Shubik, Game theory in economics: Charac- 
teristic function, core and stable set, RAND Rep. $R-904-N S F / 6$, 1973.

Straffin, P., and J. P. Heaney, Game theory and the Tennessee Valley Authority, Int. J. Game Theory, 10, 35-43, 1981.

Suzuki, M., and M. Nakayama, The cost assignment of the cooperative water resource development: A game theoretical approach, Manage. Sci., 22, 1081-1086, 1976.

Thomas, A. L., The allocation problem: Part two, in Studies in Accounting Research 9, American Accounting Association, Sarasota, Florida, 1974.

Von Neumann, J., and O. Morgenstern, Theory of Games and Economic Behavior, Princeton University Press, Princeton, New Jersey, 1944.
Water Resources Council, Policies, standards, and procedures in the formulation, evaluation, and review of plans for use and development of water and related land resources, Senate Doc. 97 , 87th Congress, 2nd Session, 1962.

Young, H. P., Cost allocation and demand revelation in public enterprises, Rep. WP-80-130, Int. Inst. for Appl. Systems Anal., Laxenburg, Austria, 1980.

Zajac, E., Fairness or Efficiency: An Introduction to Public Utility Pricing, Ballinger, Cambridge, Massachusetts, 1978.

(Received March 13, 1981;

revised November 6, 1981;

accepted December 4, 1981.) 